\title{
Intracranial and extracranial complications of acute mastoiditis: Evaluation with computed tomography
}

\author{
DEAN J. SHANLEY, CPT, MC, USA \\ THOMAS F. MURPHY, MAJ, MC, USA
}

The incidence of acute mastoiditis has decreased significantly because we can effectively treat otitis media with oral antibiotics. Inadequate or delayed treatment of otitis predisposes to the development of mastoiditis and more serious, life-threatening complications. Extension of the infectious process beyond the mastoid system can lead to a variety of intracranial and extracranial complications including meningitis, epidural and intracerebral abscesses, vascular thrombosis, osteomyelitis, and abscesses deep within the neck. Signs of clinical deterioration in a patient with otitis media should indicate to the general practitioner that a more serious condition is evolving and surgical intervention may be necessary. Computed tomography is considered the imaging modality of choice for patients with acute mastoiditis because it can define clearly the regional anatomy and provide important diagnostic information.

(Key words: Otitis media, acute mastoiditis, intracerebral abscess, meningitis, Bezold's abscess, computed tomography)

Acute mastoiditis is a serious potential complication of otitis media if the infection is left untreated or is inadequately treated. ${ }^{1}$ When first seen, the patient has fever, retroauricu-

From the Department of Radiology, Tripler Army Medical Center, Honolulu.

The opinions or assertions contained herein are the private views of the authors and are not to be construed as official or as reflecting the views of the Department of the Army or Department of Defense.

Reprint requests to Cpt Dean J. Shanley, MC, USA, Department of Radiology, Tripler Army Medical Center, Honolulu, HI 96859-5000. lar pain and swelling, and drainage from the ear if the tympanic membrane has perforated. Further progression of the infection within the middle ear spaces may lead to cholesteatoma formation, destruction of the bony ossicles, and permanent hearing loss. Coalescent mastoiditis develops as the surrounding temporal bone becomes involved in the inflammatory process, increasing the risk for intracranial extension of the infection.

Intracerebral abscesses, subdural empyemas, and meningitis are potentially life-threatening complications associated with intracranial extension of acute mastoiditis. ${ }^{2}$ Extracranial complications may also occur if the infection descends into the soft tissues of the neck. Vascular thrombosis, osteomyelitis, and abscess formation may occur as the infectious process descends along the tissue planes of the neck. ${ }^{3}$

Acute mastoiditis is currently an infrequent complication of otitis media, with most larger series reporting an incidence of fewer than 100 cases during a 10-year-period. ${ }^{4,5}$ Effective antibiotic therapy and management of otitis media are responsible for the low incidence.

The following cases are reported to illustrate the spectrum of potential complications associated with acute mastoiditis. Early evaluation with computed tomography (CT) is able to provide important diagnostic information and should be performed on all patients with clinical signs and symptoms suggesting intracranial or extracranial extension of the disease process.

\section{Report of cases}

\section{Case 1}

A 12-year-old boy with a history of chronic right 

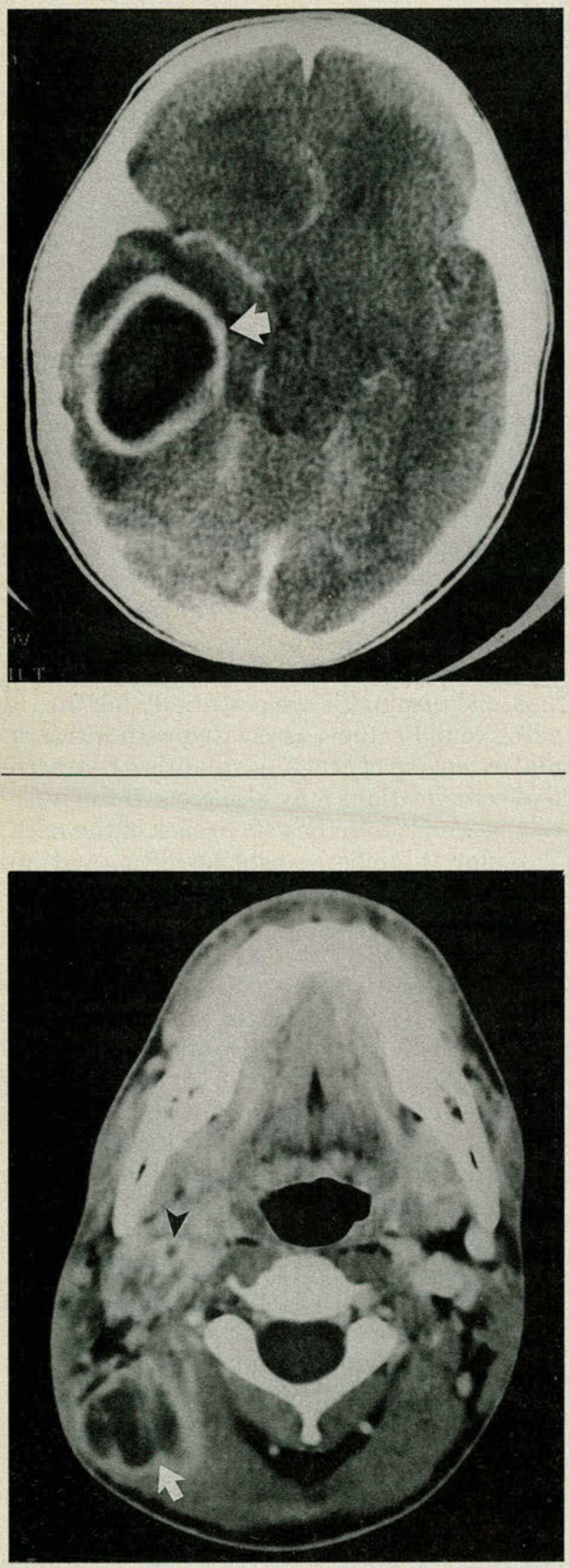

Figure 1. Intracerebral abscess (arro)w in 12-year-old boy with acute mastoiditis. Enhanced CT scan of head demonstrates low-density fluid collection with ring enhancement and surrounding edema in right temporal lobe.

otitis media was transferred to the hospital after a 2-week course of worsening headache, fever, somnolence, and gait disturbance. Purulent drainage from the right ear was present, and the right frontoparietal and temporal regions were tender to palpation. A CT scan of the head, performed on an emergency basis, revealed a large right temporal lobe abscess (Fig 1), an epidural abscess, and mastoiditis. The patient was immediately taken to the operating room for a drainage procedure. After the abscesses were evacuated, complete erosion of the incus and malleus was evident. The patient's clinical status improved greatly over the next several days, but he was left with complete hearing loss on the right. Cultures of the cerebral abscesses grew multiple anaerobes, including Bacteroides fragilis, $B$ thetaiotaomicron, Peptostreptococcus species, and diphtheroid-like species.

\section{Case 2}

A 21-year-old man was transferred to Tripler Army Hospital for evaluation of a possible neck abscess. He had a long history of recurrent right otitis media and hearing loss and was currently being treated for an episode of acute right mastoiditis without significant improvement despite intravenous antibiotic therapy. Physical examination revealed purulent drainage from the right ear and a fluctuant, tender mass in the right side of the neck. An emergency CT scan of the head and neck demonstrated an abscess in the right side of the neck (Fig 2) and destruction of the mastoid portion of the right temporal bone. Thrombosis of the right jugular vein and right sigmoid sinus was also identified (Fig 3). The patient subsequently underwent a radical mastoidectomy and drainage of the neck abscess.

Findings at surgery included a cholesteatoma within the right mastoid system and complete erosion of the ossicles. Bony dehiscence was present in the anteroinferior aspect of the mastoid tip, and

Figure 2. Deep neck abscess secondary to extracranial extension of acute mastoiditis. An axial image through neck after intravenous administration of contrast demonstrates low-density fluid collection with ring enhancement in right side of neck (arrow). Thrombosis of left internal jugular vein is also present (arrowhead). 
Figure 3. Sigmoid sinus thrombosis secondary to acute mastoiditis. Enhanced scan through posterior fossa shows area of decreased density in right sigmoid sinus (open arrow) consistent with thrombosis. Left sigmoid sinus (solid arrow) has normal pattern of enhancement.

pus extended from the mastoid tip down into the neck abscess. Multiple organisms, including Staphylococcus aureus and Bacteroides species were cultured from the abscess drainage. The patient did well postoperatively but has complete loss of hearing on the right.

\section{Case 3}

A 9-month-old girl was admitted to Tripler Army Hospital with seizures and spiking fevers of recent onset. Approximately 2 weeks before admission, she had been evaluated at a neighboring island hospital and diagnosed as having left otitis media. She had been treated with oral antibiotics and returned a week later because of increasing lethargy and persistent fevers. After lumbar puncture, Gram stain of the cerebral spinal fluid showed gramnegative cocci, suggesting Haemophilus influenza meningitis. Cultures of the spinal fluid subsequently grew out $H$ influenza. An intravenous antibiotic regimen was started but the patient continued to have fever spikes and generalized seizure activity.

After the patient was transferred to our hospital, an emergency CT scan of the head demonstrated meningeal enhancement (Fig 4) and a subdural effusion in the anterior portion of the interhemispheric fissure. The patient completed a 14day course of intravenous antibiotic therapy and gradually returned to a normal level of activity. Brainstem auditory evoked responses, elicited because hearing impairment was suspected clinically, demonstrated profound hearing loss bilaterally.

\section{Discussion}

Otitis media can be treated successfully in most cases with the oral antibiotics currently available. Patients in whom acute mastoiditis develops in spite of appropriate treatment for otitis usually will show signs of clinical dete-

Figure 4. Haemophilus influenza meningitis in a 9-monthold girl. After administration of contrast, axial image demonstrates meningeal enhancement (arrows) over cerebral convexities.
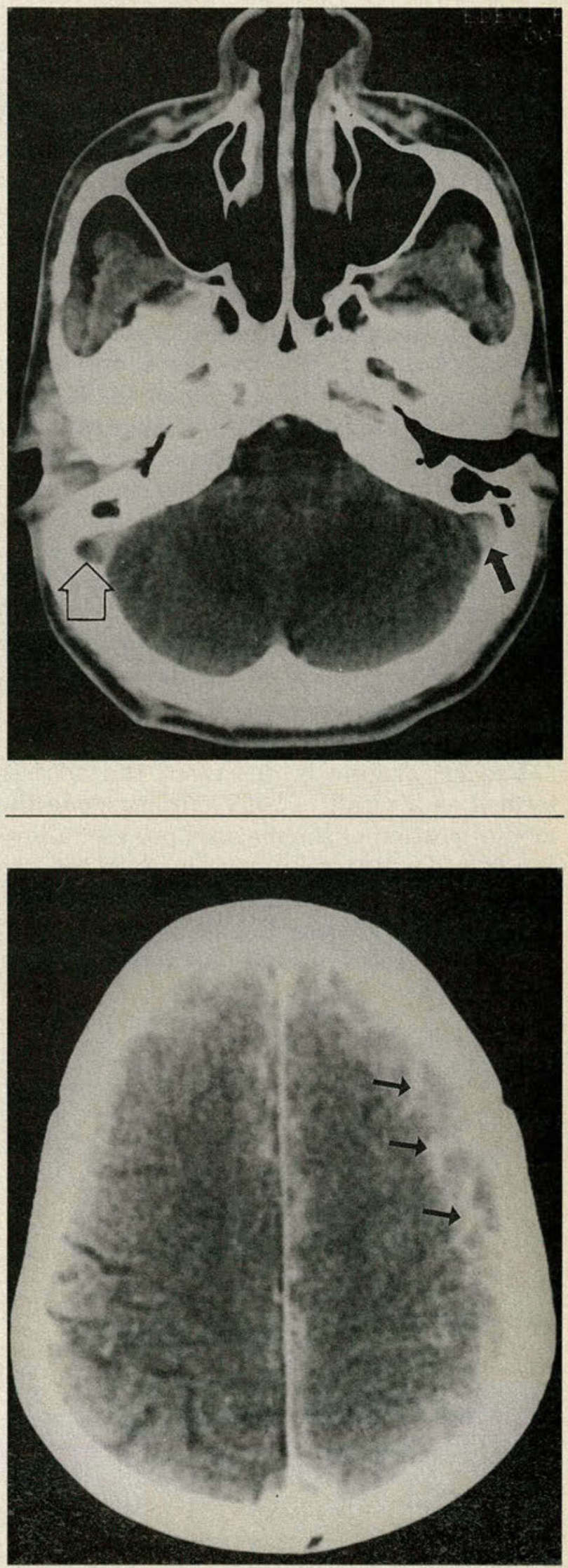
rioration or develop new signs or symptoms secondary to the spread of the infection. Seizure activity, persistent fevers, or progressive lethargy should indicate to the clinician that a more serious condition has evolved. Evaluation of the head, neck, and temporal bone region with CT is indicated for patients whose symptoms suggest intracranial or extracranial spread of the infectious process. ${ }^{6,7}$

Dehiscence of the bony roof or floor of the middle ear secondary to the inflammatory process may lead to both intracranial and extracranial complications. Erosion of the tegmen tympani, a thin plate of bone separating the middle ear from the intracranial cavity, enables the infectious process to gain access to the cranial fossae. A spectrum of complications including meningitis, venous sinus thrombosis, and epidural and intracerebral abscesses may result. Destruction of the bony floor of the middle ear and erosion through the mastoid air cells can lead to the development of a subperiosteal abscess and a zygomatic abscess.

Bezold ${ }^{8}$ originally described the abscess formed as a result of bony erosion along the medial portion of the mastoid process, allowing pus to extravasate into the deep soft tissues of the neck. This abscess now is referred to as Bezold's abscess. Following this route of spread, the extravasated pus may extend as far down as the mediastinum. Extensive involvement of the soft tissues in the neck may result in vascular thrombosis, paravertebral abscesses, or osteomyelitis of the cervical spine. Computed tomography, because of its ability to delineate the soft tissue compartments of the neck, is useful for determining the location of abscesses deep in the neck and involvement of the surrounding soft tissue and bony structures.

\section{References}

1. Ramsey PG, Weymuller EA: Complications of bacterial infection of the ears, paranasal sinuses, and oropharynx in adults. Emerg Med Clin North Am 1985;3:143-160.

2. Gower D, McGuirt WF: Intracranial complications of acute and chronic ear disease: A problem still with us. Laryngoscope 1983;93:1028-1033.

3. Venezio FR, Naidich TP, Shulman ST: Complications of mastoiditis with special emphasis on venous sinus thrombosis. $J$ Pediatr 1982;101:509-513.

4. Hawkins DB, Dru D, House JW, Clark RW: Acute mastoiditis in children: A review of 54 cases. Laryngoscope 1983;93:568572.

5. Rosen A, Ophir D, Gabriel M: Acute mastoiditis: A review of 69 cases. Ann Otol Rhinol Laryngol 1986;95:222-224.

6. Mafee MF, Singleton EL, Valvassori GE, et al: Acute otomastoiditis and its complications: Role of CT. Radiology 1985;155:391-397.

7. Zizmor J, Noyek AM: Inflammatory diseases of the temporal bone. Radiol Clin North Am 1974;12:491-504.

8. Bezold F, Siebenmann F: Textbook of otology, in Shambaugh GE, Glasscock ME (eds): Surgery of the Ear, ed 3. Philadelphia, $\mathrm{Pa}$, WB Saunders, 1980, pp 253-255. 